\title{
Multiple cranial nerve palsy revealing hypertrophic pachymeningitis with positive myeloperoxidase-antineutrophil cytoplasmic antibody
}

\author{
S. El Aoud, F. Frikha, R. Ben Salah, M. Snoussi, H. Loukil, Z. Bahloul \\ Department of Internal Medicine, Hedi Chaker Hospital, Tunisia
}

\section{SUMMARY}

Pachymeningitis is a progressive disease resulting in a diffuse thickening of dura mater due to inflammation, tumor or autoimmune diseases, but most cases are idiopathic. Here, we report the case of a 60 -year old man who had a progressive sensorineural hearing loss, visual disturbance and others cranial nerve involvement with an accompanying headache over several months. Brain magnetic resonance imaging showed diffusely thickened dura mater, highly enhanced after gadolinium administration, which was consistent with pachymeningitis. It was assumed to be related to autoimmune pathogenesis on the basis of elevated serum myeloperoxidase-antineutrophil cytoplasmic antibody titers. After empirical steroid and cyclophosphamide therapy, the neurological problems were partially improved. Therefore, in the case of atypical sensorineural hearing loss accompanied by cranial nerve palsy or headache, pachymeningitis should be considered in the differential diagnosis.

Key words: Hypertrophic pachymeningitis, Antineutrophil cytoplasmic antibodies.

Reumatismo, 2013; 65 (5): 248-252

\section{INTRODUCTION}

Dachymeningitis is a rare clinical en1 tity resulting in a diffuse thickening of dura mater. This disease has various etiologies including infection (such as syphilis or tuberculosis), neoplasm, or autoimmune disorders especially myeloperoxidase-antineutrophil cytoplasmic antibody (MPO-ANCA) positive vasculitis. When an exhaustive evaluation fails to reveal a cause, idiopathic hypertrophic cranial pachymeningitis is considered. Pachymeningitis can be complicated by cranial nerve involvement but it is rarely reported to lead to sensorineural hearing loss (1-4). Here, we present the case of a patient who had sensorineural hearing loss associated with other cranial nerve involvement revealing hypertrophic cranial pachymeningitis that was associated with positive MPO-ANCA.

Corresponding author:

Department of Internal Medicine Hedi Chaker Hospital, 3029 Sfax, Tunisia

\section{CASE REPORT}

A 60-year old man, with no medical history, was referred to our Department for pro- gressive bilateral hearing impairment, visual disturbance and anosmia. He suffered from decreased visual acuity of both eyes, pain sensation with ocular movement. $\mathrm{He}$ also complained of recurrent nonspecific headache and dry mouth syndrome over three months without dry eye syndrome.

Neurologic examination showed right facial palsy (nerve VII) and hyperesthesia of the right hemiface (nerve V). The gag reflex was missing.

An audiologic test revealed bilateral, severe mixed hearing loss with a mean threshold of $75 \mathrm{~dB}$ on the right ear and profound sensorineural hearing loss with a mean threshold of $90 \mathrm{~dB}$ on the left one in the pure tone audiogram. Ophthalmologic examination showed both peripapillary edemas that were consistent with optic neuropathy. The Schirmer test was negative. Other neurologic examinations yielded exaggerated deep tendon reflexes especially patellar and Achilles reflex with negative Babinski. The rest of the clinical examination was normal. Laboratory examination revealed that the C-reactive protein (CRP) concentration 
was markedly elevated at $110 \mathrm{mg} / \mathrm{L}(0-$ $3.0 \mathrm{mg} / \mathrm{L})$. The erythrocyte sedimentation rate (ESR) was at $120 \mathrm{~mm} \mathrm{H1}$. A complete blood picture showed white blood cell: $15,640 / \mu \mathrm{L}$, platelet: $273,000 / \mu \mathrm{L}$, eosinophilic granulocyte: $30 / \mu \mathrm{L}$ and hemoglobin: $15.6 \mathrm{~g} / \mathrm{dL}$. Protein electrophoresis with immunoglobulin, renal and liver function tests proved normal. There was no proteinuria. Cerebrospinal fluid (CSF) examination showed elevated protein level $1.66 \mathrm{~g} / \mathrm{L}(\mathrm{N}$ : $0.15-0.40), 4.24 \mathrm{mmol} / \mathrm{L}$ glucose $(\mathrm{N}: 1.5-$ 4) and 6 white cells per cubic millimeter. The virologic, parasitologic and bacteriologic analyses were negative. Carcinomatous cells were not detected in the CSF sample. Brain magnetic resonance imaging (MRI) demonstrated diffusely thickened dura mater that was hypertrophic in some sites on the left cerebral hemisphere. The T2-weighted images showed diffuse thickening of the dura mater. The meninges were highly enhanced after gadolinium administration, which was consistent with pachymeningitis (Fig. 1).

All known causes of pachymeningitis were explored. Investigations for tuberculosis, brucellosis, syphilis, Lyme disease, Epstein Barr virus, cytomegalovirus, herpes simplex virus, human immunodeficiency virus were negative. The titer of angiotensin-converting enzyme (ACE) was normal at $25 \mathrm{U}$ (Normal value: 19.8-70.2 U).

Antineutrophil cytoplasmic antibody were screened by indirect immunofluorescence technique and then tested by enzymelinked immunosorbent assay (ELISA) for circulating MPO and PR3 antibodies.

The titer of ANCA for myeloperoxidase was positive. Other autoimmune antibodies, such as, rheumatoid factor, complement activation, c-ANCA, antinuclear antibody (DNA, SSA, and SSB), anticardiolipin antibody were negative.

The focus score was inferior to 1 on the biopsy of a salivary gland according to the classification by Chisholm and Mason 1968; and there was absence of noncaseating granulomas, histiocytes, Langerhans and foreign body giant cells. A chest X-ray and chest computed tomography showed no evidence of neoplasm, sarcoidosis or re-

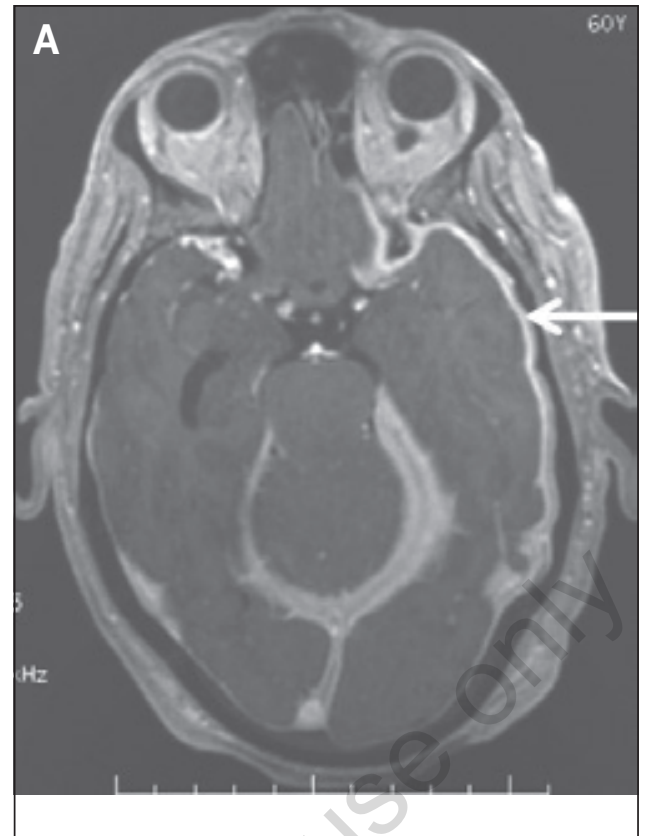

Figure 1 - Brain magnetic resonance imaging (MRI): axial (A), coronal $(B)$ and sagittal (C) T1-weighted contrast-enhanced MRI images show markedly thickened and enhanced dura extending from the vertex to the tentorium (arrow).
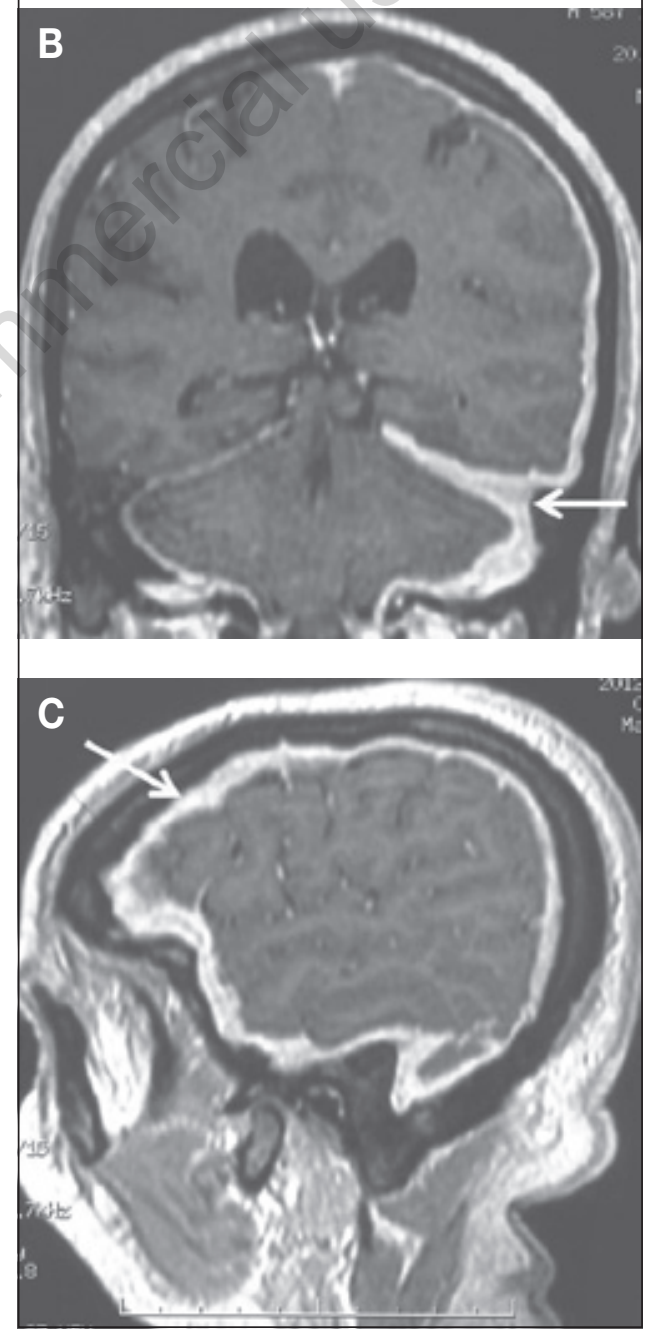
cent tuberculosis. The cause of pachymeningitis, including sarcoidosis, tuberculosis meningitis, rheumatoid arthritis, syphilis, and occult malignancy, was sought and was excluded with negative laboratory results and absence of related clinical symptoms. Though histological confirmation was not obtained, the patient was highly suspected to have cranial pachymeningitis related to autoimmune pathogenesis on the basis of elevated serum MPO-ANCA. The patient was started on oral prednisone at a dose of $60 \mathrm{mg}$ per day during two months with a progressive tapering, associated with monthly intravenous pulses of cyclophosphamide ( $1 \mathrm{~g}$ per month).

During the administration of steroids and cyclophosphamide, his headache gradually subsided. Over a 6-month treatment period, visual loss and hearing impairment gradually improved.

On repeat audiologic testing, the pure tone threshold was partially improved in the right ear $(40 \mathrm{~dB})$ more than the left one $(80 \mathrm{~dB})$ when compared with the initial pure tone audiogram. The visual problems showed a favorable response to treatment and papilledema disappeared. The ESR, the CRP and the blood regular test returned normal. The MPO-ANCA titer gradually decreased to be within the normal range.

\section{DISCUSSION}

Our patient presented with multiple cranial nerve palsy (I, II, V, VII and VIII) revealing hypertrophic pachymeningitis associated with positive MPO-ANCA, which was confirmed by neuroimaging and which improved with the association of corticosteroids and cyclophosphamid.

Pachymeningitis is a progressive rare inflammatory disease resulting in a localized or diffuse thickening of the dura mater, leptomeninges, and tentorium. Since the introduction of MRI, hypertrophic pachymeningitis has been increasingly reported in recent literature (1-8).

The clinical presentation can be heterogeneous $(7,9)$ : headache, cranial nerve palsy, cerebellar ataxia, internal carotid ar- tery occlusion, venous sinus occlusion and obstructive hydrocephalus. Other clinical presentations include diabetic insipidus with hypophysitis, and sinus thrombosis (10). In pachymeningitis, cranial nerve involvement is of concern due to high intracranial pressure resulting from thickened dura mater; or it may be directly caused by small-vessel vasculitis or indirectly by the inflammatory process of the surrounding soft tissue or of the meninges (11). Cranial nerve VIII is the most frequently involved cranial nerve $(2-4,12)$ and it represents a form of sensorineural hearing loss that is sometimes reversible. The next most frequently involved are cranial nerves V, VII, IX, X, and XII, with equal frequency (13). In most cases of pachymeningitis, optic nerve involvement is reported along with headache (14). Visual deterioration or blindness has also been described in a few reports (10).

Our patient had rapidly progressive mixed hearing loss on the right ear and sensorineural hearing loss on the left ear due to pachymeningitis. After steroid treatment associated with cyclophosphamide, the pure tone threshold improved by $35 \mathrm{~dB}$ in the right and $10 \mathrm{~dB}$ in the left ear.

Therefore, in cases of atypical sensorineural hearing loss, such as hearing loss that progresses relatively rapidly, or when neurologic signs coexist, the possibility of autoimmune pathogenesis should be taken into consideration.

The etiology of pachymeningitis is diverse and includes infectious diseases such as tuberculosis, syphilis, tumors and autoimmune disorders especially Wegener's granulomatosis (15), microscopic polyangeitis (8), relapsing polychondritis (16), rheumatoid arthritis, Sjogren's syndrome (17), sarcoidosis but mostly it is idiopathic. Recently, more research on the relationship between pachymeningitis and autoimmune diseases has been carried out. Moreover, there have been several reports about pachymeningitis with autoimmune antibody. In particular, the relationship between ANCA-positive pachymeningitis and vasculitis has been discussed $(5,6,18,19)$. In our case, the initial clinical presentation and 
subsequent remission under immunosuppressive therapy were suggestive of a vasculitis with nervous system involvement, though vasculitis was not proven histologically in this patient. Also, it was reported that the titer of MPO-ANCA decreases as the disease gradually improves through immunosuppressive treatment (12). Hence, it is suggested that an MPO-ANCA titer can be used as a monitoring marker of the disease's activity in such patients $(12,20)$.

The diagnosis of neurosarcoidosis can be difficult when neurological involvement is the first or only manifestation of the disease. In our patient, ACE level was normal, serum calcium, chest X-rays and the biopsy were normal and ruled out sarcoidosis.

To diagnose pachymeningitis, MRI with gadolinium enhancement is the most sensitive diagnostic imaging modality, and shows the relationship between the inflammatory degree of the lesion and the clinical outcome, as well as the prognosis $(1,12$, 21). Generally, in MRI, T1-weighted images show iso- or hypo-intense thickened dura mater, and $\mathrm{T} 2$-weighted images show hypointense dura mater of fibrous reacting tissue surrounded by a thin hyperintense border attributed to the acute inflammatory process. Distinctively, meninges are highly enhanced after a contrast injection $(12,21)$. Although almost cases of hypertrophic pachymeningitis appear responsive to corticosteroid therapy, some patients require combined therapy with corticosteroid and other immunosuppressive drugs (cyclophosphamide, azathioprine, methotrexate) because of the severity of symptoms as seen in the present case; or in case of progressive disease despite treatment.

Among 33 patients with idiopathic cranial hypertrophic meningitis, 62, 6\% experienced full remission without steroid dependence, $15 \%$ experienced steroid dependent partial or complete remission, $15 \%$ experienced a progressive course inspite of steroid therapy, and $32 \%$ died regardless of treatment (9).

In our patient, we chose combination of corticosteroid and intravenous pulses of cyclophosphamid (1 g per month). We noted a spectacular improvement within 6 months. There is no consensus about the duration of this therapy. After minimum 12 months we will try to stop the cyclophosphamid after brain MRI control.

\section{CONCLUSIONS}

In the case of hearing impairment accompanying other cranial neuropathy, pachymeningitis should be taken into consideration, and brain MRI with gadolinium enhancement is the best method of detecting it.

Though vasculitis was not proven histologically in our patient, we believe that MPOANCA-related autoimmunity provoked the patient's disease as already reported in similar cases. So, a cautious evaluation including an ANCA work-up should be performed. We conclude that early recognition of an autoimmune etiology, even in the absence of previous pulmonary or renal involvement, is required to ensure proper treatment and prevent definitive neurological impairment.

Conflict of interest: the authors have no conflict of interest.

Competing interest: all authors contributed equally.

\section{REFERENCES}

1. Kupersmith MJ, Martin V, Heller G, Shah A, Mitnick HJ. Idiopathic hypertrophic pachymeningitis. Neurology. 2004; 62: 686-94.

2. Oghalai JS, Ramirez AL, Hegarty JL, Jackler RK. Chronic pachymeningitis presenting as asymmetric sensorineural hearing loss. Otol Neurotol. 2004; 25: 616-21.

3. Iwasaki S, Matsui Y, Ito K, Naito R, Abbey K. Hypertrophic cranial pachymeningitis presenting as steroid-responsive hearing loss. Ann Otol Rhinol Laryngol. 2003; 112: 476-9.

4. Bovo R, Berto A, Palma S, Ceruti S, Martini A. Symmetric sensorineural progressive hearing loss from chronic idiopathic pachymeningitis. Int J Audiol. 2007; 46: 107-10.

5. Zhao P, Zhang BS. Clinical analyses of perinuclear antineutrophil cytoplasmic antibody associated hypertrophicpachymeningitis. Zhonghua Yi Xue Za Zhi. 2013; 93: 837-40.

6. Watanabe K, Tani Y, Kimura H, Asai J, Tana- 
ka K, Hayashi Y, et al. Hypertrophic cranial pachymeningitis in MPO-ANCA-related vasculitis: a case report and literature review. $\mathrm{Fu}-$ kushima J Med Sci. 2013; 59: 56-62.

7. Khalil M, Ebner F, Fazekas F, Enzinger C. Neurological picture. Idiopathic hypertrophic cranial pachymeningitis: a rare but treatable cause of headache and facial pain. J Neurol Neurosurg Psychiatry. 2013; 84: 354-5.

8. Hayashi Y, Sugawara H, Otsuka M, Yamada S, Tabei K, Ueki A. Fatal hemoperitoneum preceded by cranial hypertrophic pachymeningitis in a patient with ANCA-positive microscopic polyangitis. Inter Med. 2008; 47: 1061-3.

9. Parney IF, Johnson ES, Allen PB. Idiopathic cranial hypertrophic pachymeningitis responsiveness to antitubercular therapy. A case therapy. Neurosurgery. 1997; 41: 965-71.

10. Lee YC, Chueng YC, Hsu SW, Lui CC. Idiopathic hypertrophic cranial pachymeningitis: case report with 7 years of imaging follow-up. AJNR Am J Neuroradiol. 2003; 24: 119-23.

11. Rojana-udomsart A, Pulkes T, Viranuwatti K, Laothamatas J, Phudhichareonrat S, Witoonpanich R. Idiopathic hypertrophic cranial pachymeningitis. J Clin Neurosci. 2008; 15: 465-9.

12. Lim EJ, Kim SH, Lee SH, Lee KY, Choi JH, Nam EJ, et al. Reversible sensorineural hearing loss due to pachymeningitis associated with elevated serum MPO-ANCA. Clin Exp Otorhinolaryngol. 2011; 4: 155-8.

13. Mamelak AN, Kelly WM, Davis RL, Rosenblum ML. Idiopathic hypertrophic cranial pachymeningitis, report of three cases. J Neurosurg. 1993; 79: 270-6.

14. Hayashi S, Sugeno N, Nishiyama S, Hasegawa $\mathrm{T}$, Aoki M. Unusual visual impairments in a case of MPO-ANCA associated hypertrophic pachymeningitis. Rinsho Shinkeigaku. 2012; 52: $152-5$.

15. Just SA, Knudsen JB, Nielsen MK, Junker $\mathrm{P}$. Wegener's granulomatosis presenting with pachymeningitis: clinical and imaging remission by rituximab. ISRN Rheumatol. 2011; 2011: 608942.

16. Cañas CA, Díaz-Martínez JC, Tobón JJ. Combination of hypertrophic pachymeningitis, PR3-ANCA-positive vasculitis and relapsing polychondritis. J Rheumatol 2011; 38; 966-7.

17. Abouzaid CH, Kissani N, Essaadouni L. Cranial pachymeningitis and primary Sjögren's syndrome. Revue Neurologique. 2011; 167: 348-51.

18. Nagashima T, Maguchi S, Terayama Y, Horimoto $M$, Nemoto $M$, Nunomura $M$, et al. P-ANCA-positive Wegener's granulomatosis presenting with hypertrophic pachymeningitis and multiple cranial neuropathies: case report and review of literature. Neuropathology. 2000; 20: 23-30.

19. Fam AG, Lavine E, Lee L, Perez-Ordonez B, Goyal M. Cranial pachymeningitis: an unusual manifestation of Wegener's granulomatosis. J Rheumatol. 2003; 30: 2070-4.

20. Takagi D, Nakamaru Y, Maguchi S, Furuta Y, Fukuda S. Clinical features of bilateral progressive hearing loss associated with myeloperoxidase-antineutrophil cytoplasmic antibody. Ann Otol Rhinol Laryngol. 2004; 113: 388-93.

21. Hatano N, Behari S, Nagatani T, Kimura M, Ooka K, Saito K, et al. Idiopathic hypertrophic cranial pachymeningitis: clinicoradiological spectrum and therapeutic options. Neurosurgery. 1999; 45: 1336-42. 